\title{
Effect of Cryopreservation on Semen Sample
}

\author{
Lt Col SK Rath*, Surg Capt (Mrs) P Tarneja (Retd)+, Maj S Man Singh"
}

\begin{abstract}
There are varied indications of preserving a semen sample for utilization at a later day. However, the freeze-thaw cycle is bound to impart deleterious effect on the fertilizing potential of the sample. 60 semen samples divided into three groups were preserved in this study. On subsequent thawing, after three months it was seen that the yield varied between $66 \%$ to $72 \%$. The post thaw percent yield bears a linear relationship with the pre-freeze quality. 5 pregnancies were achieved in the study testifying the preservation of fertilizing ability of the thawed samples. Sub normal semen samples which might be otherwise acceptable in a therapeutic insemination, do not withstand freezing.
\end{abstract}

MJAFI 2004; 60 : $42-44$

Key Words : Cryopreservation, Semen

\section{Introduction}

$\mathrm{I}^{\mathrm{n}}$ 1866, Montegazza from Italy predicted that scientists of future will be able to improve breeds of horses and cattle without having to spend enormous sums of money in transporting thorough bred stallions and bulls. It will be possible to carry out insemination with frozen sperm sent from one place to another. It should be possible for a soldier dying in the battle field to have legitimate children long after. This vision about freezing semen while retaining its fertilizing potential must have been remarkable considering lack of supporting experimental evidence at his time.

The life pattern in recent times has led to an increase in infertility related problems. The quality of semen samples has shown a downward trend. Migration to greener pastures has led to the unique problem of spouse not being available at the fertile phase. In Armed Forces, separation is also compounded by stress and strain in a hard terrain. Some of these problems can be resolved by the use of the technique of cryopreservation of the semen for patients enrolled in the infertility clinic.

Donor insemination is the commonest indication for cryopreservation. Donor insemination for achieving a pregnancy has become an accepted means of therapy. However, recognition of the risk of transmission of HIV, Hepatitis B and other diseases associated with use of fresh donor semen is an unacceptable risk. Hence, it is essential that sequential screening of donors is continued pending release of cryopreserved semen for therapeutic use [1].

Though the above advantage is convincing enough to use cryopreserved semen sample in donor insemination programme, it may not be possible to preserve all available samples. It is necessary to look at the yield after thawing in one's own laboratory. The over all percent variation between pre-freeze and post thaw parameter can give an idea about setting the qualitative requirement of a sample which would be acceptable for preservation. With this background, it was decided to study the effect of cryopreservation on semen parameters at our infertility centre so that the resources could be optimally utilized.

\section{Material and Methods}

20 males from each of the following group were recruited for the study.

Males with proven fertility willing to be donor (Group A)

Male partners of infertile couples with normal semen parameters (Group B)

Male partners of infertile couples with subnormal semen parameters (Group C)

Semen was collected after masturbation directly into sterile specimen beakers made of nontoxic plastic ware (Cryogenic India). The semen samples were kept at room temperature for liquefaction to occur. On completion of liquefaction the sample was mixed well and a small quantity was taken for microscopic examination in Makler sperm counting chamber (Sefi, Israel). A single observer evaluated all samples to avoid interobserver variation in the study. The parameters assessed were total volume, colour, viscosity, liquefaction time, count, motility, morphology and pus cells. The motility was assessed as per the WHO classification i.e. rapid progressive motility, slow or sluggish motility, non progressive motility and immotile spermatozoa.

The cryoprotectant used was a buffered solution of glycerol 5\% along with egg yolk as extender and gentamycin as antibiotic. This was added to the ejaculate slowly in a drop wise fashion to avoid the damaging effects due to volume changes in the spermatozoon. The cryoprotectant was added in 1:1 ratio and left for a minimum period of 10 minutes to

${ }^{*}$ Classified Specialist (Obstetrics and Gynaecology), 151 Base Hospital, C/o 99 APO, ${ }^{+}$Ex- Professor and Head, Department of Obstetrics and Gynaecology, Armed Forces Medical College, Pune - 411 040, "Graded Specialist (Obstetrics and Gynaecology), 92 Base Hospital, C/o 56 APO. 
Table 1

Mean value of various semen parameters

\begin{tabular}{|c|c|c|c|c|c|c|c|c|c|c|c|}
\hline \multirow{3}{*}{ Group } & \multirow{3}{*}{$\begin{array}{c}\text { Mean } \\
\text { volume } \\
\text { in } \mathrm{ml}\end{array}$} & \multirow{2}{*}{\multicolumn{2}{|c|}{$\begin{array}{l}\text { Count in } \\
\text { million/ml }\end{array}$}} & \multicolumn{4}{|c|}{ Motility in $\%$} & \multicolumn{4}{|c|}{ Normal morphology in $\%$} \\
\hline & & & & \multicolumn{2}{|c|}{ Pre freeze } & \multicolumn{2}{|c|}{ Post thaw } & \multicolumn{2}{|c|}{ Pre freeze } & \multicolumn{2}{|c|}{ Post thaw } \\
\hline & & Mean & SD & Mean & $\overline{\mathrm{SD}}$ & $\overline{\text { Mean }}$ & $\overline{\mathrm{SD}}$ & $\overline{\text { Mean }}$ & $\overline{\mathrm{SD}}$ & $\overline{\text { Mean }}$ & $\overline{\mathrm{SD}}$ \\
\hline A & 3.525 & 120 & 22.606 & 66.25 & 7.232 & 48.25 & 7.31 & 71.5 & 4.89 & 64 & 4.167 \\
\hline B & 3.325 & 84 & 19.574 & 66 & 5.982 & 46.5 & 6.71 & 70 & 5.12 & 58.25 & 4.941 \\
\hline $\mathrm{C}$ & 3.4 & 14.75 & 5.003 & 48.75 & 11.22 & 32.25 & 8.65 & 50.75 & 7.82 & 38.25 & 8.925 \\
\hline
\end{tabular}

allow the spermatozoon to complete the volume changes. All samples that were to be cryo preserved were kept in vials in aliquots of one $\mathrm{ml}$ and numbered uniquely for identification at a later date without any ambiguity. The vials were held in vial holders which in turn were kept in a canister. The canister was lowered in two stages into liquid nitrogen kept in cryocan (Fig 1). Initially they were suspended in the liquid nitrogen vapours for 15 minutes to allow initial slow rate freezing. Then they were dipped into liquid nitrogen. After $24 \mathrm{hrs}$ one of the ampoules was checked for cryosurvivability and only if the first thawed sample contained motile sperms it was accepted. Otherwise all the samples of the batch were discarded.

The donor was always tested for HIV, HBsAg and VDRL and the sperm samples were kept under quarantine in the sperm bank for a period of 3 months. The donor was again tested for HIV, HBsAg and VDRL. If the second test was negative, the stored samples were released for intra-uterine insemination (IUI) programmes. All treatment cycles for this study had been stimulated with Clomiphene Citrate 100mg OD from day 2 to day 5 of the cycle. The cycles were

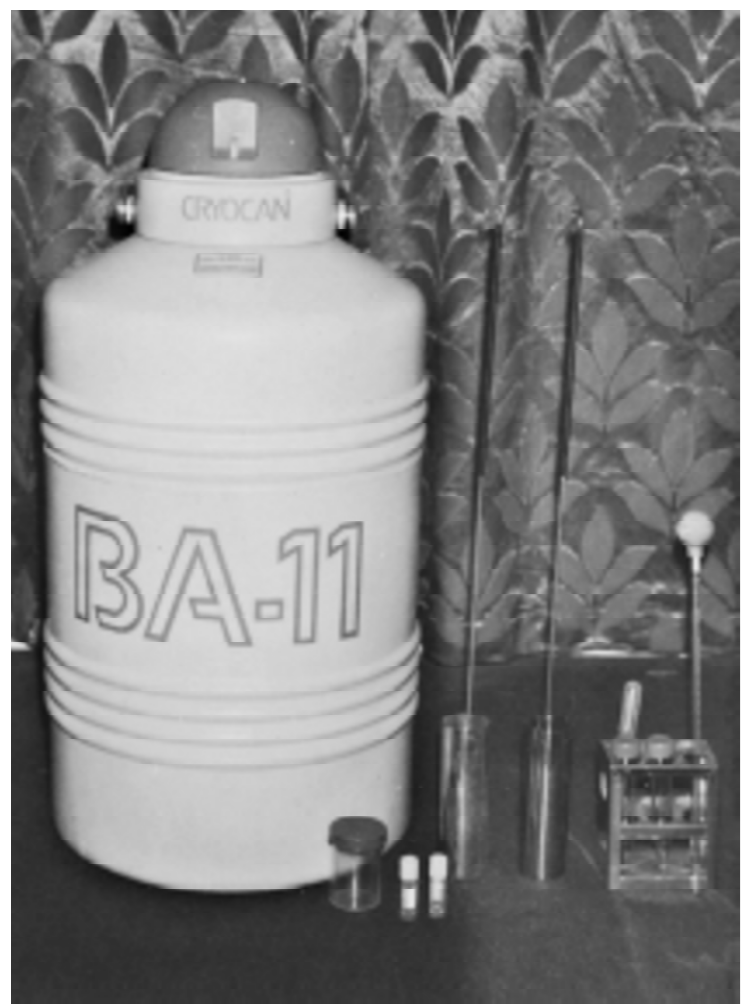

Fig. 1 : Equipment for cryopreservation-cryocan, semen beaker, cryovial and cannister monitored with trans vaginal sonography. For IUI, the desired ampoule was removed and thawed by keeping at room temperature for 15-20 minutes. After this the sample was incubated at $37^{\circ} \mathrm{C}$ for 30 minutes. The post thaw parameters of motility, viability and morphology were checked and the results were entered in the proforma. The parameters of pre freeze and post thaw samples were compared. This was followed by usual swim up techniques of preparation for insemination. In case of a missed period following IUI, urine was tested for pregnancy.

\section{Results}

60 samples were frozen in the study, 20 samples each from the group A, B and C. At the first check for viability 24 hours after freeze, 2 samples from group $\mathrm{C}$ were discarded. No case tested positive for HIV, HBs Ag or VDRL after the quarantine period. 17 samples from group A, 15 from group B and 10 from group $\mathrm{C}$ were used as and when indicated. The mean value of the important parameters of pre freeze and post thaw after 3 months of preservation that would determine the fertilizing potential are given in Table 1 .

The percent variation and yield percent of motility/ morphology was calculated from the following formulae and summarized in Table 2.

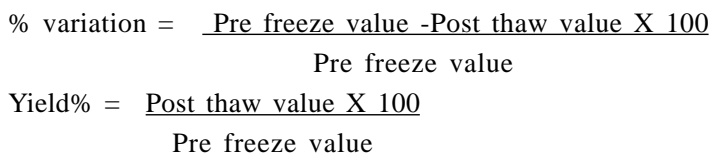

3 pregnancies with Group A samples and 2 pregnancies with group B samples were achieved. There were no pregnancies with Group C samples.

\section{Discussion}

The British Andrology Society recommends screening semen donor for sexually transmitted infections to minimize the risk of pathogen transmission to the mother and fetus. The American Fertility Society and the Centers for Disease Control USA, have recommended the use of only cryopreserved, quarantined donor semen for therapeutic insemination, in order to safeguard against possibility of transmission of infectious diseases [2,3].

Cryopreservation of semen can be done by conventional method used in this study which achieves approximately a reduction of temperature of $-10^{\circ} \mathrm{C} /$ minute in the vapour phase. Other methods include rapid freezing at $37^{\circ} \mathrm{C} /$ minute by holding the ampoules on a 
Table 2

Variation and yield in post thaw sample

\begin{tabular}{lccccc} 
Group & \multicolumn{2}{c}{ Motility } & & \multicolumn{2}{c}{ Morphology } \\
\cline { 2 - 3 } \cline { 5 - 6 } \% variation & \% yield & & \% variation & \% yield \\
A & 27.17 & 72.83 & & 10.49 & 89.51 \\
B & 29.55 & 70.45 & & 16.78 & 83.22 \\
C & 33.75 & 66.15 & & 24.63 & 75.37
\end{tabular}

platform $3 \mathrm{~cm}$ above the liquid phase [4]. In programmed freezing, the temperature is reduced from ambient temperature at the rate of $-1^{\circ} \mathrm{C} /$ minute upto $5^{\circ} \mathrm{C}$ followed by $-10^{\circ} \mathrm{C} /$ minute till $-85^{\circ} \mathrm{C}$. After this the straws are plunged into liquid nitrogen at $-196^{\circ} \mathrm{C}$. Semi programmed freezing techniques are also in use. The cryopreservation and subsequent thawing invariably decreases the sperm motility during these procedures [5]. The phase transition encountered by the plasma membrane during the freeze thaw cycle makes the membrane more prone to early fracture at stress points [6]. Programmed freezing techniques are said to be less variable and expose the samples to a uniform rate. The yield after thawing such samples is better as compared to two stage freezing used in this study [7]. However, the cost of equipment for vapour freezing technique used in this study is cheaper.

The percent variation and yield for Group A and B are close to each other and comparable to the results obtained by other workers [8]. However, the report by Mossad showed that the percent variation is significantly more between two such groups [9]. It is worth noting in this context that the yield rates were generally lower in their study and could be method related. Our results are also comparable with that of Trummer who worked with similar sample size [10].

An interesting feature of that study is the influence of cryopreservation temperature. They found that though the optimum cryopreservation temperature is $196^{\circ} \mathrm{C}, 28 \%$ motility can be preserved even at $-70^{\circ} \mathrm{C}$. The conception rate of $17.5 \%$ and $13 \%$ obtained with single insemination with group A \& B respectively in our study is acceptable. When the semen sample is subnormal the deleterious effects are more pronounced, as shown by a poor yield and higher variation in post thaw samples of Group C. 10 samples were used from this group for insemination of those couples who did not wish to exercise the option of donor insemination. However, no pregnancy resulted. Pooling of samples to increase the sperm concentration for such cases has been reported by Kobayashi [11]. It can be concluded that the post thaw yield in cryopreservation is directly proportional to the initial sperm concentration and discretion has to be exercised in accepting a sample for freezing.

\section{References}

1. Craig JM, Barratt CL, Kinghorn GR. Semen donors and STD screening. Genitourin Med 1997,73(4):280-3.

2. American Fertility Society. Revised new guidelines for the use of semen in donor insemination. Fertility and Sterility 1988;49:211-2.

3. American Fertility Society. Ethical considerations of the new reproductive technologies. Fertility and Sterility 1990;53(Suppl 2):1-87.

4. Kaneka S, Lee H, Kobayashi T, Oda T, Izuka R. Cryo preservation of washed and concentrated human sperm and its application to AIH. Journal of Fertilization and Implantation 1990;7:120-3.

5. Sharma R, Vemulapalli S, Kohn S, Agarwal A. Effect of centrifuge speed refrigeration medium and sperm washing medium on cryopreserved semen quality after thawing. Arch Androl 1997;39(1):33-8.

6. Alvarez JG, Storey BT. Evidence that membrane stress contribute more than lipid peroxidation to cryodamage during preservation of human sperm. J Androl 1993;14:199-209.

7. Check ML, Check DJ, Katsoff D, Check JK. Improved results of thawed sperm cryo preserved with slow stage cooling with a cellevator. Arch Androl 1996;37(1): 61-4.

8. Polaez TE, Stronk J, Xiong C, Jones EE, Olive DL, Huszar G. Optimal utilization of human semen for assisted reproduction : recovery and maintenace of sperm motility and viability. $\mathrm{J}$ Assist Reprod Genet 1998;15(8):504-12.

9. Mossad H, Morsedi M, Toner JP, Oehninger S. Impact of cryopreservation on spermatozoa from infertile men, implication for artificial inseminaiton. Arch Androl 1994;33(1):51-7.

10. Trummer H, Tucker K, Young C, Kaul N, Mcacham RB. Effect of storage temperature on sperm cryopreservation. Fertil Steril 1998;70(6):1162-4.

11. Kobayashi T, Kaneko S, Hara I, Park YJ, Sato H. Concentrating human sperm before cryopreservation. Andrologia 1991;23(1):25-8.

Outside of a dog, a book is man's best friend. Inside of a dog, it's too dark to read. 